\title{
The Cohomology Rings of Some $p$-Groups
}

By

\author{
Angelina CHIN *
}

\begin{abstract}
We determine the mod- $p$ cohomology ring and some of the integral cohomology ring structure of a $p$-group expressible as an extension with kernel cyclic of order $p$ and quotient $C_{p^{m}} \oplus C_{p^{n}}$, where $m, n>1$.
\end{abstract}

\section{$\S 1$. Introduction}

Let $p$ denote an odd prime and let $P_{m, n}$ be the group with presentation of the form

$$
\left\langle A, B, C \mid A^{p^{m}}=B^{p^{n}}=C^{p}=[A, C]=[B, C]=1,[A, B]=C\right\rangle,
$$

where $m, n \geq 1$. We may express $P_{m, n}$ as a central extension of the form

$$
1 \rightarrow \mathrm{C} \rightarrow P_{m, n} \rightarrow L \rightarrow 1
$$

where $\mathbf{C}=\langle C\rangle \cong C_{p}$ and $L=\langle\bar{A}, \bar{B}\rangle \cong C_{p^{m}} \times C_{p^{n}}$. In this paper we shall determine the mod- $p$ cohomology ring and some of the integral cohomology ring structure of $P_{m, n}$ when $m, n>1$. For the case when $m=n=1, P_{1,1}$ is the non-abelian group of order $p^{3}$ and exponent $p$, and the integral and mod- $p$ cohomology rings of $P_{1,1}$ are known (see [4], [5], [7]). We note that for $p=3$ and $m, n>1$, Leary in [6] has obtained the Poincaré series of $H^{*}\left(P_{m, n}, \mathbb{F}_{3}\right)$.

Let $G=P_{m, n}$ where $m, n>1$. It is clear that we may assume, without loss of generality, that $m \geq n \geq 2$. In section 2 of this paper we shall review some facts on Massey products. Then in section 3, we shall use Massey products to define some generators of degree two in the mod-p cohomology ring of $G$ and to determine some of the relations involving these generators. This explicit use of Massey products to obtain the cohomology ring structure has been demonstrated by Leary in [3] and [5]. We remark here that the mod-p cohomology ring structure of $G$

Communicated by Y. Ihara, March 9, 1995.

1991 Mathematics Subject Classification: 20J06.

* Department of Mathematics, The University of Queensland, St. Lucia, QLD 4072, Australia. 
turns out to be less complicated than that for the case $m=n=1$ (see Theorem 3.1).

It is more difficult to determine the integral cohomology ring of $G$. In section 4 of this paper we shall use the mod-p cohomology ring of $G$ obtained in section 3 to determine the additive structure of the $\operatorname{ring} H^{*}(G, \mathbb{Z})$ and some of its multiplicative relations.

\section{$\S 2$. Massey Products}

In $[3,5]$, Leary showed how Massey products can be used to define some generators of low degrees in a mod- $p$ cohomology ring and to obtain some of the relations involving these generators. Leary went on to show in [6] that the image of the differential $d_{4}$ in the Lyndon-Hochschild-Serre (LHS) spectral sequence (with $\mathbb{F}_{p}$ coefficients) of certain central extensions involves a Massey product. Since then, Clark ([2]) used matric Massey products, which are generalisations of Massey products, to determine the mod-2 cohomology ring of the group $U_{3}(4)$.

In this section we shall review the definition and some properties of Massey products. Most of what follows in this section can be found in [3].

Let $R$ be a commutative ring with identity on which $G$ acts trivially ( $G$ here is an arbitrary finite group). For $u \in H^{*}(G, R)$, we write $(-1)^{u}$ for $(-1)^{\operatorname{deg} u}$. Let $\mathbf{P}=\left(P^{*}, \boldsymbol{\partial}\right)$ be the standard or bar resolution of $\mathbb{F}_{p}$ over $\mathbb{F}_{p} G$ and let $\mathbb{C}=\left(C^{*}, \boldsymbol{\delta}\right)$ be the cochain complex where $C^{*}=\operatorname{Hom}_{F_{p} G}\left(P^{*}, R\right)$. Let $[u],[v]$ and $[w]$ denote elements in $H^{*}(G, R)$ represented by $u, v$ and $w$, respectively. If [uv] $=0$ and $[v w]=0$ in $H^{*}(G, R)$, then there are elements $a, b$ in the cochain complex $\mathbf{C}$ with

$$
\delta(a)=u v \text { and } \delta(b)=v w .
$$

The Massey product of $[u],[v]$ and $[w]$ written $\langle[u],[v],[w]\rangle$ is then defined as

$$
\begin{aligned}
\langle[u],[v],[w]\rangle & =\left[(-1)^{u} u b-a w\right] \\
& \in H^{u+v+w-1}(G, R) /\left(u H^{v+w-1}(G, R)+w H^{u+v-1}(G, R)\right) .
\end{aligned}
$$

It is straightforward to verify that the Massey product is trilinear.

The following properties are satisfied by Massey products, whenever all the terms are defined, for any $u, v, w, x, y \in H^{*}(G, R)$ :

(i) $\langle u, v, w\rangle x+(-1)^{u} u\langle v, w, x\rangle \equiv 0 \bmod u H^{*} x$;

(ii) $(-1)^{u}\langle\langle u, v, w\rangle, x, y\rangle+\langle u,\langle v, w, x\rangle, y\rangle+(-1)^{u}\langle u, v,\langle w, x, y\rangle\rangle \equiv 0$ $\bmod u H^{*}+H^{u+v-1} w H^{x+y-1}+y H^{*}$

(iii) $(-1)^{w u}\langle u, v, w\rangle+(-1)^{u v}\langle v, w, u\rangle+(-1)^{v w}\langle w, u, v\rangle \equiv 0$

$$
\bmod u H^{*}+v H^{*}+w H^{*}
$$


(iv) $\langle u, v, w\rangle+(-1)^{u v+v w+w u}\langle w, v, u\rangle \equiv 0 \bmod u H^{*}+w H^{*}$.

Let $\Delta: H^{2}\left(G, \mathbb{F}_{p}\right) \rightarrow H^{\imath+1}\left(G, \mathbb{F}_{p}\right)$ be the mod-p Bockstein. The following result is well-known to the experts already. A proof of it using the bar resolution can be found in $[3]$.

Lemma 2.1. Let $p>2$ be a prime and let $x$ generate $H^{1}\left(G_{p}, \mathbb{F}_{p}\right)$. Then $\langle x, x, x\rangle$ is a unique element of $H^{2}\left(G_{p}, \mathbb{F}_{p}\right)$ and

$$
\langle x, x, x\rangle=\left\{\begin{array}{ll}
0 & \text { if } p>3 \\
\Delta(x) & \text { if } p=3
\end{array}\right. \text {. }
$$

We are now ready to determine the mod-p cohomology ring of $G$.

\section{§3. The Ring Structure of $H^{*}\left(G, \mathbb{F}_{p}\right)$}

Consider the LHS spectral sequence for extension (e) with coefficients in $\mathbb{F}_{p}$. Since $\mathbf{C}$ is central in $G$, so $L$ acts trivially on $H^{*}\left(\mathbf{C}, \mathbb{F}_{p}\right)$. It follows from the universal coefficient theorem that the $E_{2}$-term of the spectral sequence is given by

$$
\begin{aligned}
E_{2}^{* * *} & =H^{*}\left(L, H^{*}\left(\mathbf{C}, \mathbb{F}_{p}\right)\right) \\
& \cong H^{*}\left(L, \mathbb{F}_{p}\right) \otimes H^{*}\left(\mathbf{C}, \mathbb{F}_{p}\right) \\
& \cong \Lambda\left[x_{1}, x_{2}, u\right] \otimes \mathbb{F}_{p}\left[y_{1}, y_{2}, v\right],
\end{aligned}
$$

where $\operatorname{deg} x_{1}=\operatorname{deg} x_{2}=\operatorname{deg} u=1, \operatorname{deg} y_{1}=\operatorname{deg} y_{2}=\operatorname{deg} v=2, \Delta\left(x_{1}\right)=\Delta\left(x_{2}\right)=$ 0 and $\Delta u=v$. Since $H^{1}\left(G, \mathbb{F}_{p}\right) \cong \operatorname{Hom}\left(G, \mathbb{F}_{p}\right) \cong \mathbb{Z}_{p} \oplus \mathbb{Z}_{p}$, it follows by degree reasons that $x_{1}, x_{2} \in E_{2}^{1.0}$ survive to $E_{\infty}$ and the differential $d_{2}$ must kill the extension class That is, $d_{2}(u)=\lambda x_{1} x_{2}$ for some $\lambda \not \equiv 0(\bmod p)$. Therefore, $d_{2}\left(x_{1} u\right)=d_{2}\left(x_{2} u\right)=0$. Since $d_{2}(v)=0$, it follows that the $E_{3}$-page of the LHS spectral sequence is generated by the elements $x_{1}, x_{2} \in E_{3}^{1,0}, y_{1}, y_{2} \in E_{3}^{2,0}, v \in E_{3}^{0,2}$ and $x_{1} u, x_{2} u \in E_{3}^{1,1}$ subject to the relations

$$
\begin{gathered}
x_{1}^{2}=x_{2}^{2}=x_{1} x_{2}=0,\left(x_{1} u\right)^{2}=\left(x_{2} u\right)^{2}=\left(x_{1} u\right)\left(x_{2} u\right)=0, \\
x_{1}\left(x_{2} u\right)=-x_{2}\left(x_{1} u\right), x_{1}\left(x_{1} u\right)=x_{2}\left(x_{2} u\right)=0 .
\end{gathered}
$$

Now since Bockstein commutes with transgressions, we have that

$$
d_{3}(v)=d_{3}(\Delta(u))=\Delta\left(d_{2}(u)\right)=\Delta\left(\lambda x_{1} x_{2}\right)=0 .
$$

For degree reasons, it follows that $d_{\imath}=0$ for $i>3$. Therefore the spectral sequence collapses at $E_{3}$, that is, $E_{\infty}=E_{3}$. 
Now consider the bigraded Poincare series $P_{3}\left(t, t^{\prime}\right)$ of the $E_{3}$-page of the spectral sequence, that is,

$$
P_{3}\left(t, t^{\prime}\right)=\sum_{i, j} t^{i} t^{\prime j} \operatorname{dim}_{\mathbb{F}_{p}} E_{3}^{i, j}
$$

We have

$$
P_{3}(t, t \prime)=\frac{1+2 t+2 t t \prime+t^{2} t \prime}{\left(1-t^{2}\right)^{2}\left(1-t \prime^{2}\right)}
$$

Then since the spectral sequence collapses at $E_{3}$, it follows that the Poincare series of $H^{*}\left(G, \mathbb{F}_{p}\right)$ is

$$
P_{G}(t)=P_{3}(t, t)=\frac{1+2 t+2 t^{2}+t^{3}}{\left(1-t^{2}\right)^{3}}
$$

By abuse of notation, let $x_{1}, x_{2}, y_{1}, y_{2}$ and $v$ denote the generators in the ring $H^{*}\left(G, \mathbb{F}_{p}\right)$ which correspond to the generators of the same name in $E_{3}=E_{\infty}$. Clearly, the relation $x_{1}^{2}=x_{2}^{2}=x_{1} x_{2}=0$ holds in the ring $H^{*}\left(G, \mathbb{F}_{p}\right)$. Because of these relations we may define unique elements in $H^{2}\left(G, \mathbb{F}_{p}\right)$ by forming the Massey product of any three elements of $H^{1}\left(G, \mathbb{F}_{p}\right)$. Since $m, n>1$, it follows from Lemma 2.1 that $\left\langle x_{1}, x_{1}, x_{1}\right\rangle=\left\langle x_{2}, x_{2}, x_{2}\right\rangle=0$. Let $Y_{1}=\left\langle x_{1}, x_{1}, x_{2}\right\rangle$ and $Y_{2}=\left\langle x_{2}, x_{2}, x_{1}\right\rangle$. By using the same argument as in [3, Lemma 2.13] we can show that $y_{1}, y_{2}, Y_{1}, Y_{2}$ and $v$ are independent elements of $H^{2}\left(G, \mathbb{F}_{p}\right)$. Then since $\operatorname{dim}_{\mathbb{F}_{p}}$ $H^{2}\left(G, \mathbb{F}_{p}\right)=5$, it follows that the elements $y_{1}, y_{2}, Y_{1}, Y_{2}$ and $v$ form a basis for $H^{2}\left(G, \mathbb{F}_{p}\right)$.

Now from the identities satisfied by Massey products, we have

$$
\begin{aligned}
x_{1} Y_{2} & =x_{1}\left\langle x_{2}, x_{2}, x_{1}\right\rangle \\
& \equiv x_{1}\left\langle x_{1}, x_{2}, x_{2}\right\rangle \bmod x_{1}\left(x_{2} H^{1}\left(G, \mathbb{F}_{p}\right)+x_{1} H^{1}\left(G, \mathbb{F}_{p}\right)\right)=0 \\
& \equiv\left\langle x_{1}, x_{1}, x_{2}\right\rangle x_{2} \bmod x_{1} H^{1}\left(G, \mathbb{F}_{p}\right) x_{2}=0 \\
& =Y_{1} x_{2}=x_{2} Y_{1}, \\
x_{1} Y_{1} & =x_{1}\left\langle x_{1}, x_{1}, x_{2}\right\rangle \\
& \equiv\left\langle x_{1}, x_{1}, x_{1}\right\rangle x_{2} \bmod x_{1} H^{1}\left(G, \mathbb{F}_{p}\right) x_{2}=0 \\
& =0
\end{aligned}
$$

and

$$
\begin{aligned}
x_{2} Y_{2} & =x_{2}\left\langle x_{2}, x_{2}, x_{1}\right\rangle \\
& \equiv\left\langle x_{2}, x_{2}, x_{2}\right\rangle x_{1} \bmod x_{2} H^{1}\left(G, \mathbb{F}_{p}\right) x_{1}=0 \\
& =0 .
\end{aligned}
$$


From the Poincaré series of $H^{*}\left(G, \mathbb{F}_{p}\right)$ we have that $\operatorname{dim}_{\mathbb{F}_{p}} H^{4}\left(G, \mathbb{F}_{p}\right)=12$. By inspection, $H^{4}\left(G, \mathbb{F}_{p}\right)$ has basis

$$
\left\{y_{1}^{2}, y_{1} y_{2}, y_{2}^{2}, y_{1} Y_{1}, y_{1} Y_{2}, y_{2} Y_{1}, y_{2} Y_{2}, y_{1} v, y_{2} v, Y_{1} v, Y_{2} v, v^{2}\right\}
$$

Consider the subspace of $H^{4}\left(G, \mathbb{F}_{p}\right)$ which restricts trivially to $\langle A, C\rangle$ and $\langle B, C\rangle$. This subspace contains $Y_{1}^{2}, Y_{2}^{2}, Y_{1} Y_{2}$ and has basis $\left\{y_{1} y_{2}, y_{1} Y_{2}, y_{2} Y_{1}\right\}$. Therefore there are expressions of the form

$$
\begin{gathered}
Y_{1}^{2}=a_{1} y_{1} y_{2}+a_{2} y_{1} Y_{2}+a_{3} y_{2} Y_{1} \\
Y_{2}^{2}=b_{1} y_{1} y_{2}+b_{2} y_{1} Y_{2}+b_{3} y_{2} Y_{1} \\
Y_{1} Y_{2}=c_{1} y_{1} y_{2}+c_{2} y_{1} Y_{2}+c_{3} y_{2} Y_{1}
\end{gathered}
$$

for some $a_{i}, b_{i}, c_{i} \in \mathbb{Z}(i=1,2,3)$.

Taking the product of (3.1) with $x_{1}$ and making use of the fact that $x_{1} y_{1} y_{2}$ and $x_{1} y_{1} Y_{2}$ are $\mathbb{F}_{p}$-linearly independent, it follows that $a_{1}, a_{2} \equiv 0(\bmod p)$. Therefore, $Y_{1}^{2}=a_{3} y_{2} Y_{1}$. By taking the product of the last equation with $x_{2}$ we have $0=$ $a_{3} x_{2} y_{2} Y_{1}$. It follows that $a_{3} \equiv 0(\bmod p)$ and hence, $Y_{1}^{2}=0$. By the same argument we can show that $Y_{2}^{2}=Y_{1} Y_{2}=0$.

Now consider the graded $\mathbb{F}_{p}$-subalgebra $S=\sum_{i \geq 0} S_{i}$ of $H^{*}\left(G, \mathbb{F}_{p}\right)$ generated by the elements $x_{1}, x_{2}, y_{1}, y_{2}, Y_{1}, Y_{2}$ and $v$ as above. It is clear that $S$ is free and finitely generated over the polynomial subring $\mathbb{F}_{p}\left[y_{1}, y_{2}, v\right]$ with generators $1, x_{1}, x_{2}, Y_{1}, Y_{2}$ and $x_{1} Y_{2}$. We then have

$$
\sum_{i \geq 0} t^{i} \operatorname{dim}_{\mathbb{F}_{p}} S_{\imath}=\frac{1+2 t+2 t^{2}+t^{3}}{\left(1-t^{2}\right)^{3}}=P_{G}(t) .
$$

It follows that $H^{*}\left(G, \mathbb{F}_{p}\right) \cong S$ as $\mathbb{F}_{p}$-algebras. We have therefore proved

Theorem 3.1. Let $G=\langle A, B, C| A^{p^{m}}=B^{p^{n}}=C^{p}=[A, C]=[B, C]=1$, $[A, B]=C\rangle$, where $m, n>1$. Then the mod-p cohomology ring $H^{*}\left(G, \mathbb{F}_{p}\right)$ is generated as an $\mathbb{F}_{p}$-algebra by the elements

$$
x_{1}, x_{2}, y_{1}, y_{2}, Y_{1}, Y_{2}, v
$$

where

$$
\operatorname{deg} x_{1}=\operatorname{deg} x_{2}=1, \operatorname{deg} y_{1}=\operatorname{deg} y_{2}=\operatorname{deg} Y_{1}=\operatorname{deg} Y_{2}=\operatorname{deg} v=2
$$

subject to the relations 


$$
\begin{gathered}
x_{1}^{2}=x_{2}^{2}=x_{1} x_{2}=0, x_{1} Y_{2}=x_{2} Y_{1}, x_{1} Y_{1}=x_{2} Y_{2}=0, \\
Y_{1}^{2}=Y_{2}^{2}=Y_{1} Y_{2}=0 .
\end{gathered}
$$

We remark that Theorem 3.1 tells us that for a fixed prime $p$, there are infinitely many non-isomorphic non-abelian $p$-groups with isomorphic mod-p cohomology rings.

\section{§4. The Ring Structure of $H^{*}(G, \mathbb{Z})$}

We begin with a general result on $\operatorname{dim}_{\mathbb{F}_{p}}\left(H^{i}(G, \mathbb{Z}) \otimes_{\mathbb{Z}} \mathbb{F}_{p}\right)$ for $i>0$. Assume for the moment that $G$ is an arbitrary finite group. Let $Q(t)=\Sigma_{\imath \geq 0} t^{i} \operatorname{dim}_{F_{p}}$ $\left(H^{i}(G, \mathbb{Z}) \otimes_{\mathbb{Z}} \mathbb{F}_{p}\right)$ and $P(t)=\sum_{i \geq 0} t^{i} \operatorname{dim}_{\mathbb{F}_{p}} H^{i}\left(G, \mathbb{F}_{p}\right)$. That is, $P(t)$ is the Poincare series of $H^{*}\left(G, \mathbb{F}_{p}\right)$. The following relation between $P(t)$ and $Q(t)$ has been proven in $[1]$. We give a proof here for the sake of completeness.

\section{Lemma 4.1.}

$$
Q(t)=\frac{t}{1+t} P(t)+\frac{1}{1+t} .
$$

Proof. Let $H^{i}(G, \mathbb{Z})_{p}$ denote the $p$-component of $H^{i}(G, \mathbb{Z})$. Consider the long exact sequence in cohomology

$$
\begin{aligned}
\ldots \longrightarrow H^{i}(G, \mathbb{Z})_{p} \stackrel{p^{(\imath)}}{\longrightarrow} H^{i}(G, \mathbb{Z})_{p} \stackrel{\pi_{*}^{(i)}}{\longrightarrow} H^{i}\left(G, \mathbb{F}_{p}\right) \\
\stackrel{\delta^{(i)}}{\longrightarrow} H^{i+1}(G, \mathbb{Z})_{p} \longrightarrow \ldots
\end{aligned}
$$

which is induced from the short exact sequence $0 \longrightarrow \mathbb{Z} \stackrel{p}{\longrightarrow} \mathbb{Z} \stackrel{\pi}{\longrightarrow} \mathbb{F}_{p} \longrightarrow 0$. Note that

$$
\operatorname{Im} \pi_{*}^{(i)} \cong H^{i}(G, \mathbb{Z})_{p} / \operatorname{Im} p^{(i)} \cong H^{i}(G, \mathbb{Z}) \otimes_{\mathbb{Z}} \mathbb{F}_{p}
$$

and

$$
\operatorname{Im} \delta^{(i)} \cong H^{i}\left(G, \mathbb{F}_{p}\right) / \operatorname{Im} \pi_{*}^{(i)}
$$

We also note that $\operatorname{Im} \delta^{(i)}=\operatorname{Ker} p^{(i+1)}={ }_{p} H^{i+1}(G, \mathbb{Z})$ and $\operatorname{dim}_{\mathbb{F}_{p} p} H^{i+1}(G, \mathbb{Z})=\operatorname{dim}_{\mathbb{F}_{p}}$ $H^{i+1}(G, \mathbb{Z}) \otimes_{\mathbb{Z}} \mathbb{F}_{p}$, where ${ }_{p} H^{i+1}(G, \mathbb{Z})=\left\{x \in H^{i+1}(G, \mathbb{Z}): p x=0\right\}$. We therefore have

$$
\operatorname{dim}_{\mathbb{F}_{p}} H^{l+1}(G, \mathbb{Z}) \otimes_{\mathbb{Z}} \mathbb{F}_{p}=\operatorname{dim}_{\mathbb{F}_{p}} H^{i}\left(G, \mathbb{F}_{p}\right)-\operatorname{dim}_{\mathbb{F}_{p}} H^{i}(G, \mathbb{Z}) \otimes_{\mathbb{Z}} \mathbb{F}_{p}
$$


It follows from this that

$$
\begin{aligned}
P(t)-Q(t) & =\frac{1}{t}\left(Q(t)-\operatorname{dim}_{\mathbb{F}_{p}}\left(H^{0}(G, \mathbb{Z}) \otimes_{\mathbb{Z}} \mathbb{F}_{p}\right)\right) \\
& =\frac{1}{t}(Q(t)-1) .
\end{aligned}
$$

Therefore, $Q(t)=\frac{t}{1+t} P(t)+\frac{1}{1+t}$.

For a commutative ring $R$, let $R\left\{a_{1}, \ldots, a_{k}\right\}$ denote the free $R$-module with generators $a_{1}, \ldots, a_{k}$. Now let $G$ be the $p$-group as defined at the beginning of the paper.

Lemma 4.2. Let $P_{k}$ be the coefficient of $t^{k}$ in $P_{G}(t)$. Then

$$
P_{2 r}=\frac{3 r^{2}+5 r+2}{2}(r \geq 0)
$$

and

$$
P_{2 r-1}=\frac{3 r^{2}+r}{2}(r \geq 1)
$$

Proof. Consider the graded $\mathbb{F}_{p}$-algebra

$$
S=\mathbb{F}_{p}\left[y_{1}, y_{2}, v\right]\left\{1, x_{1}, x_{2}, Y_{1}, Y_{2}, x_{1} Y_{2}\right\}
$$

as defined in section 3 . Let $T_{2 r}$ be the dimension over $\mathbb{F}_{p}$ of the $r$ th symmetric power of the polynomial algebra $\mathbb{F}_{p}\left[y_{1}, y_{2}, v\right\rfloor$ and let $T(t)=\Sigma_{r \geq 0} T_{2 r} t^{2 r}$. Then $T(t)=\frac{1}{\left(1-t^{2}\right)^{3}}$ and by computing we have

$$
T_{2 r}=1+\cdots+(r+1)=\frac{(r+1)(r+2)}{2}, r \geq 0
$$

By Theorem 3.1 we have that $P_{0}=1$ and $P_{1}=2$. We have shown in section 3 that $H^{*}\left(G, \mathbb{F}_{p}\right) \cong S$ (as $\mathbb{F}_{p}$-algebras). Then since $\operatorname{deg} Y_{1}=\operatorname{deg} Y_{2}=2$, it follows that

$$
P_{2 r}=T_{2 r}+2 T_{2 r-2}=\frac{3 r^{2}+5 r+2}{2}, r \geq 1 \text {. }
$$

Since deg $x_{1}=\operatorname{deg} x_{2}=1$ and $\operatorname{deg} x_{1} Y_{2}=3$, we also have that

$$
P_{2 r-1}=2 T_{2 r-2}+T_{2 r-4}=\frac{3 r^{2}+r}{2}, r \geq 2
$$

Lemma 4.3. Let $Q_{k}$ be the coefficient of $t^{k}$ in $Q(t)$. Then 


$$
Q_{2 r+1}=r^{2}+2 r(r \geq 0)
$$

and

$$
Q_{2 r}=\frac{r^{2}+r+2}{2}(r \geq 0)
$$

Proof. Since $\frac{1}{1+t}=1-t+t^{2}-t^{3}+t^{4}-t^{5}+\ldots$, it follows from Lemma 4.1 that

$$
\begin{aligned}
& Q_{0}=1, \quad Q_{1}=0, \quad Q_{2}=P_{1}=2, \\
& \begin{aligned}
Q_{2 r+1} & =\left(P_{2 r}+P_{2 r-2}+\cdots+P_{2}+1\right)-\left(P_{2 r-1}+P_{2 r-3}+\cdots+P_{1}\right)-1 \\
& =\sum_{k=1}^{r} \frac{3 k^{2}+5 k+2}{2}-\sum_{k=1}^{r} \frac{3 k^{2}+k}{2} \\
& =r^{2}+2 r(r \geq 1)
\end{aligned}
\end{aligned}
$$

and

$$
\begin{aligned}
Q_{2 r} & =\left(P_{2 r-1}+P_{2 r-3}+\cdots+P_{1}\right)-\left(P_{2 r-2}+P_{2 r-4}+\cdots+P_{2}+1\right)+1 \\
& =\sum_{k=1}^{r} \frac{3 k^{2}+k}{2}-\sum_{k=1}^{r-1} \frac{3 k^{2}+5 k+2}{2} \\
& =\frac{r^{2}+r+2}{2}(r \geq 2) .
\end{aligned}
$$

Using the argument in [7, Proposition 4.3], we can generalise the same result in [7] to the following:

Proposition 4.4. For any odd prime $p$ and any positive integer's $r$, such that $r \geq s \geq 1$,

$$
H^{*}\left(C_{p^{r}} \times C_{p^{s}}, \mathbb{Z}\right) \cong P[\alpha, \beta] \otimes \Lambda[\eta]
$$

where $\operatorname{deg} \alpha=\operatorname{deg} \beta=2$ and $\operatorname{deg} \eta=3$ with relations

$$
p^{r} \alpha=p^{s} \beta=p^{s} \eta=0, \quad \eta^{2}=0
$$

We note that $H^{2}(G, \mathbb{Z}) \cong \operatorname{Hom}(G, \mathbb{Q} / \mathbb{Z}) \cong \mathbb{Z}_{p^{m}} \oplus \mathbb{Z}_{p^{n}}$. Now consider the LHS spectral sequence for extension (e) with coefficients in $\mathbb{Z}$. The $E_{2}$-term of the LHS spectral sequence for this extension is given by

$$
E_{2}^{i, j}=H^{i}\left(L, H^{i}(\mathbf{C}, \mathbb{Z})\right), i, j \geq 0
$$

Since $H^{\text {odd }}(\mathbf{C}, \mathbb{Z})=0$, we have that $d_{2 i}=0$ for $i \geq 1$. Therefore $E_{2 i} \cong E_{2 i+1}$ for 
$i \geq 1$. By Proposition 4.4 we have that $H^{*}(L, \mathbb{Z}) \cong P[\alpha, \beta] \otimes \Lambda[\eta]$ where $\operatorname{deg} \alpha=$ $\operatorname{deg} \beta=2$ and $\operatorname{deg} \eta=3$ such that $p^{m} \alpha=p^{n} \beta=p^{n} \eta=0$. We then note that $E_{2}^{2,0} \cong H^{2}(L, \mathbb{Z}) \cong \mathbb{Z}_{p^{m}} \alpha \oplus \mathbb{Z}_{p^{n}} \beta, E_{2}^{1,1}=0$ and $E_{2}^{0,2}=H^{2}(\mathbf{C}, \mathbb{Z}) \cong \mathbb{Z}_{p} \gamma$. For degree reasons, $\alpha$ and $\beta$ must survive to $E_{\infty}$. Since $H^{2}(G, \mathbb{Z})$ has order $p^{m+n}$, so $\gamma \in E_{2}^{0,2}$ cannot survive to $E_{\infty}$. Since $p \gamma=0$ and $p^{n} \eta=0$, it follows by degree reasons that $d_{3}(\gamma)=s p^{n-1} \eta$ for some $s \not \equiv 0(\bmod p)$. We thus have that $\eta \in E_{2}^{3,0} \cong H^{3}(L, \mathbb{Z})$ survives to $E_{\infty}$ with $p^{n-1} \operatorname{Inf}_{L, G} \eta=0$. Note that $E_{2}^{2,1}=E_{2}^{0,3}=0$ and $E_{2}^{1,2} \cong H^{1}\left(L, \mathbb{F}_{p}\right)$ $\cong \mathbb{Z}_{p} \mu \oplus \mathbb{Z}_{p} \nu$. Since the coefficient of $t^{3}$ in $Q(t)$ is 3 (by Lemma 4.3), so $\mu$ and $\nu$ must survive to $E_{\infty}$. By abuse of notation we therefore have that $H^{3}(G, \mathbb{Z}) \cong$ $\mathbb{Z}_{p^{n-1}} \eta \oplus \mathbb{Z}_{p} \mu \oplus \mathbb{Z}_{p} \nu$.

We next note that $E_{2}^{4,0} \cong H^{4}(L, \mathbb{Z}) \cong \mathbb{Z}_{p^{m}} \alpha^{2} \oplus \mathbb{Z}_{p^{n}} \alpha \beta \oplus \mathbb{Z}_{p^{n}} \beta^{2}, E_{2}^{3,1}=E_{2}^{1,3}=0$, $E_{2}^{2,2} \cong H^{2}\left(L, \mathbb{F}_{p}\right) \cong \mathbb{Z}_{p} \alpha \gamma \oplus \mathbb{Z}_{p} \beta \gamma \oplus \mathbb{Z}_{p} \chi$ and $E_{2}^{0,4}=H^{4}(\mathbf{C}, \mathbb{Z}) \cong \mathbb{Z}_{p} \gamma^{2}$. Since $d_{3}(\alpha \gamma)=$ $s p^{n-1} \alpha \eta \neq 0$ and $d_{3}(\beta \gamma)=s p^{n-1} \beta \eta \neq 0$, it follows that $\alpha \gamma, \beta \gamma \in E_{2}^{2,2}$ do not survive to $E_{\infty}$. Since $E_{2}^{5,0} \cong H^{5}(L, \mathbb{Z}) \cong \mathbb{Z}_{p^{n}} \alpha \eta \oplus \mathbb{Z}_{p^{n}} \beta \eta$, we see that $d_{3}(\chi+a \alpha \gamma+b \beta \gamma)=0$ for some $a, b \in \mathbb{Z}$. Then for degree reasons and by abusing notation if necessary, we have that $E_{\infty}^{2,2} \cong \mathbb{Z}_{p} \chi$.

By inspection, we have the structure of $E_{2}$ as follows:

\section{Lemma 4.5.}

$$
\begin{aligned}
& E_{2}^{2 r, 0} \cong \mathbb{Z}_{p^{m}} \alpha^{r} \oplus\left(\oplus_{i+\jmath=r-1} \mathbb{Z}_{p^{n}} \alpha^{i} \beta^{j+1}\right)(r \geq 1) \\
& E_{2}^{1,0}=0 ; \\
& E_{2}^{2 r+1,0} \cong \oplus_{i+j=r-1} \mathbb{Z}_{p^{n}} \alpha^{i} \beta^{j} \eta(r \geq 1) ; \\
& E_{2}^{*, 2 s+1}=0(s \geq 0) ; \\
& E_{2}^{0,2 s} \cong \mathbb{Z}_{p} \gamma^{s}(s \geq 1) ; \\
& E_{2}^{2 r+1,2 s} \cong \oplus_{i+j=r}\left(\mathbb{Z}_{p} \alpha^{i} \beta^{j} \mu \gamma^{s-1} \oplus \mathbb{Z}_{p} \alpha^{i} \beta^{j} \nu \gamma^{s-1}\right)(r \geq 0, s \geq 1) ; \\
& E_{2}^{2 r, 2 s} \cong\left(\oplus_{i+j=r} \mathbb{Z}_{p} \alpha^{2} \beta^{j} \gamma^{s}\right) \oplus\left(\oplus_{i+j}=r-1 \mathbb{Z}_{p} \alpha^{i} \beta^{j} \chi \gamma^{s-1}\right)(r, s \geq 1)
\end{aligned}
$$

Proposition 4.6. Every generator of the group $E_{2}^{i, j}$, where $i+j$ is odd, survives to $E_{\infty}$.

Proof. By Lemma 4.5 we have that the number of independent generators of $E_{2}^{i, j}$, where $i+j=2 r+1(r \geq 1)$, is

$$
r+2(1+\cdots+r)=r+\frac{2 r(r+1)}{2}=r^{2}+2 r
$$

By Lemma 4.3 we have that $Q_{2 r+1}=r^{2}+2 r, r \geq 1$. It follows from this that all the generators of $E_{2}^{i, j}$, where $i+j$ is odd, must survive to $E_{\infty}$. 
Proposition 4.7. The subring of $H^{*}(G, \mathbb{Z})$ generated by $\alpha$ and $\beta$ is isomorphic to $\mathbb{Z}[\alpha, \beta] /\left(p^{m} \alpha, p^{n} \beta\right)$.

Proof. Since $d_{2 i}=0$ for $i \geq 1$, we see that the only possible way that an element in $E_{2}^{2 j, 0}(j \geq 2)$ does not survive to $E_{\infty}$ is if one of the differentials

$$
d_{2 i-1}: E_{2 i-1}^{2 j-2 i+1,2 i-2} \rightarrow E_{2 i-1}^{2 j, 0}, \quad i \geq 2
$$

is non-zero. But since all the generators of $E_{2}^{2 j-2 i+1,2 i-2}$ survive to $E_{\infty}$ (by Proposition $4.6)$, it follows that no non-zero element of $E_{2 i-1}^{2 j .0}(i \geq 2)$ can be hit by any of the differentials in the spectral sequence. We thus have that the subring generated by $\alpha$ and $\beta$ in $H^{*}(G, \mathbb{Z})$ is a polynomial subring with $p^{m} \alpha=p^{n} \beta=0$.

Next we consider the element $\gamma^{2} \in E_{2}^{0,4}$. Since $d_{3}\left(\gamma^{2}\right)=2 \gamma\left(s p^{n-1} \eta\right)=0$, so $\gamma^{2} \in E_{2}^{0,4} \cong E_{3}^{0,4}$ survives to $E_{4}$. We have to determine if $d_{5}\left(\gamma^{2}\right)=0$. First consider the case $n=2$. Since $p \alpha \eta, p \beta \eta \in \operatorname{Im} d_{3}$, we have that $E_{4}^{5,0} \cong \mathbb{Z}_{p} \alpha \eta \oplus \mathbb{Z}_{p} \beta \eta$. By Proposition 4.6 all the generators of $\oplus_{i+j=5} E_{2}^{i, j}$ must survive to $E_{\infty}$. In particular, $\alpha \eta$ and $\beta \eta$ in $E_{2}^{5,0}$ survive to $E_{\infty}$. It follows that $d_{5}\left(\gamma^{2}\right)$ must be zero and hence, $\gamma^{2}$ survives to $E_{\infty}$. Now consider the case $n \geq 3$. Let

$$
q: P_{m, n} \rightarrow P_{m, n} /\left\langle A^{p^{2}}, B^{p^{2}}\right\rangle \cong P_{2,2}
$$

be the quotient map and consider the following induced diagram of central extensions:

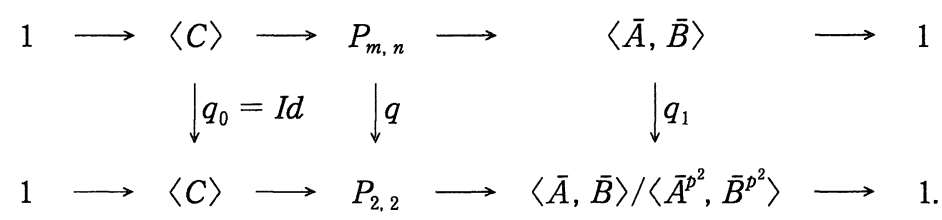

Let $q^{*}: E_{r}^{* * *}(2,2) \rightarrow E_{r}^{* * *}(m, n)$ be the induced map of spectral sequences. Since $d_{5}\left(\gamma^{2}\right)=0$ in $E_{r}^{* * *}(2,2)$, it follows that

$$
0=q^{*} d_{5}\left(\gamma^{2}\right)=d_{5}\left(q_{0}^{*} \gamma^{2}\right)=d_{5}\left(\gamma^{2}\right)
$$

in $E_{r}^{* * *}(m, n)$. We therefore have that $\gamma^{2} \in E_{2}^{0,4}$ survives to $E_{\infty}$ for all $n \geq 2$. It follows that $\oplus_{i+\jmath=4} E_{\infty}^{i, j} \cong \mathbb{Z}_{p^{m}} \alpha^{2} \oplus \mathbb{Z}_{p^{n}} \alpha \beta \oplus \mathbb{Z}_{p^{n}} \beta^{2} \oplus \mathbb{Z}_{p} \chi \oplus \mathbb{Z}_{p} \gamma^{2}$ and hence, $H^{4}(G, \mathbb{Z})$ has order $p^{m+2 n+2}$. Since the coefficient of $t^{4}$ in $Q(t)$ is 4 (by Lemma 4.3), we must have

$$
H^{4}(G, \mathbb{Z}) \cong \mathbb{Z}_{p^{m}} \alpha^{2} \oplus \mathbb{Z}_{p^{n}} \alpha \beta \oplus \mathbb{Z}_{p^{n}} \beta^{2} \oplus \mathbb{Z}_{p^{2} \xi}
$$


where we may take $\xi$ such that $\operatorname{Res}_{G, c} \xi=\gamma^{2}$. Since all the generators of $\oplus_{\imath+j=5} E_{2}^{\imath, j}$ survive to $E_{\infty}$ (by Proposition 4.6), we have that

$$
\begin{gathered}
H^{5}(G, \mathbb{Z}) \cong \mathbb{Z}_{p^{n-1}} \alpha \eta \oplus \mathbb{Z}_{p^{n-1}} \beta \eta \oplus \mathbb{Z}_{p} \alpha \mu \oplus \mathbb{Z}_{p} \alpha \nu \oplus \mathbb{Z}_{p} \beta \mu \oplus \mathbb{Z}_{p} \beta \nu \\
\oplus \mathbb{Z}_{p} \varepsilon_{1} \oplus \mathbb{Z}_{p} \varepsilon_{2},
\end{gathered}
$$

where $\varepsilon_{1}, \varepsilon_{2}$ correspond to $\mu \gamma, \nu \gamma \in E_{2}^{1,4}$, respectively.

Next, by Lemma 4.5 we have that $E_{2}^{6,0} \cong \mathbb{Z}_{p^{m}} \alpha^{3} \oplus \mathbb{Z}_{p^{n}} \alpha^{2} \beta \oplus \mathbb{Z}_{p^{n}} \alpha \beta^{2} \oplus \mathbb{Z}_{p^{n}} \beta^{3}$, $E_{2}^{5,1}=E_{2}^{3,3}=E_{2}^{1,5}=0, E_{2}^{4,2} \cong \mathbb{Z}_{p} \alpha^{2} \gamma \oplus \mathbb{Z}_{p} \alpha \beta \gamma \oplus \mathbb{Z}_{p} \beta^{2} \gamma \oplus \mathbb{Z}_{p} \alpha \chi \oplus \mathbb{Z}_{p} \beta \chi, E_{2}^{2,4} \cong \mathbb{Z}_{p} \alpha \gamma^{2} \oplus$ $\mathbb{Z}_{p} \beta \gamma^{2} \oplus \mathbb{Z}_{p} \chi \gamma$ and $E_{2}^{0,6} \cong \mathbb{Z}_{p} \gamma^{3}$. Note that $d_{3}\left(\alpha^{2} \gamma\right)=s p^{n-1} \alpha^{2} \eta \neq 0, d_{3}(\alpha \beta \gamma)=s p^{n-1} \alpha \beta \eta$ $\neq 0$ and $d_{3}\left(\beta^{2} \gamma\right)=s p^{n-1} \beta^{2} \eta \neq 0$. Therefore, $\alpha^{2} \gamma, \alpha \beta \gamma$ and $\beta^{2} \gamma$ in $E_{3}^{4,2}$ do not survive to $E_{\infty}$ and $E_{4}^{7,0} \cong \mathbb{Z}_{p^{n-1}} \alpha^{2} \eta \oplus \mathbb{Z}_{p^{n-1}} \alpha \beta \eta \oplus \mathbb{Z}_{p^{n-1}} \beta^{2} \eta$. Since all the generators of $\oplus_{i+j=7} E_{2}^{i, j}$ must survive to $E_{\infty}$ (by Proposition 4.6), it is clear that the elements $\chi \gamma \in E_{2}^{2.4}$ and $\gamma^{3} \in E_{2}^{0,6}$ must survive to $E_{\infty}$ if $n=2$. By using the same argument as for the element $\gamma^{2} \in E_{2}^{0.4}$, we can show that the elements $\chi \gamma \in E_{2}^{2,4}$ and $\gamma^{3} \in E_{2}^{0,6}$ also survive to $E_{\infty}$ if $n \geq 3$. Therefore $H^{6}(G, \mathbb{Z})$ has order $p^{m+3 n+6}$. Then since the coefficient of $t^{6}$ in $Q(t)$ is 7 (by Lemma 4.3), there must exist a generator $\zeta \in H^{6}(G, \mathbb{Z})$ such that

$$
H^{6}(G, \mathbb{Z}) \cong \mathbb{Z}_{p^{m}} \alpha^{3} \oplus \mathbb{Z}_{p^{n}} \alpha^{2} \beta \oplus \mathbb{Z}_{p^{n}} \alpha \beta^{2} \oplus \mathbb{Z}_{p^{n}} \beta^{3} \oplus \mathbb{Z}_{p^{2}} \alpha \xi \oplus \mathbb{Z}_{p^{2}} \beta \xi \oplus \mathbb{Z}_{p^{2}} \zeta
$$

Clearly, we may take $\zeta \in H^{6}(G, \mathbb{Z})$ such that $\operatorname{Res}_{G, \mathrm{c}} \zeta=\gamma^{3}$. By inspection we have the structure of $E_{4}$ as follows:

\section{Lemma 4.8.}

$$
\begin{aligned}
& E_{4}^{2 r, 0} \cong \mathbb{Z}_{p^{m}} \alpha^{r} \oplus\left(\oplus_{\imath+\jmath=r-1} \mathbb{Z}_{p^{n}} \alpha^{2} \beta^{\jmath+1}\right)(r \geq 1) ; \\
& E_{4}^{1,0}=0 \text {; } \\
& E_{4}^{2 r+1,0} \cong \oplus_{i+j=r-1} \mathbb{Z}_{p^{n-1}} \alpha^{2} \beta^{j} \eta(r \geq 1) \text {; } \\
& E_{4}^{* 2 s+1}=0(s \geq 0) \text {; } \\
& E_{4}^{2 r+1,2 s} \cong \oplus_{\imath+\jmath}=r\left(\mathbb{Z}_{p} \alpha^{2} \beta^{\jmath} \mu \gamma^{s-1} \oplus \mathbb{Z}_{p} \alpha^{2} \beta^{\jmath} \nu \gamma^{s-1}\right)(r \geq 0, s \geq 1) ; \\
& E_{4}^{2 r, 2} \cong \oplus_{\imath+j=r-1} \mathbb{Z}_{p} \alpha^{2} \beta^{\jmath} \chi(r \geq 1) ; \\
& E_{4}^{2 r, 4 s} \cong\left(\oplus_{i+\jmath=r} \mathbb{Z}_{p} \alpha^{2} \beta^{j} \gamma^{2 s}\right) \oplus\left(\oplus_{i+\jmath=r-1} \mathbb{Z}_{p} \alpha^{i} \beta^{j} \chi \gamma^{2 s-1}\right)(r, s \geq 1) ; \\
& E_{4}^{2 r, 4 s+2} \cong\left(\oplus_{\imath+\jmath=r} \mathbb{Z}_{p} \alpha^{i} \beta^{j} \gamma^{2 s+1}\right) \oplus\left(\oplus_{\imath+\jmath=r-1} \mathbb{Z}_{p} \alpha^{2} \beta^{j} \chi \gamma^{2 s}\right)(r, s \geq 1) ; \\
& E_{4}^{0,2}=0 \text {; } \\
& E_{4}^{0,4 s} \cong \mathbb{Z}_{p} \gamma^{2 s}(s \geq 1) \\
& E_{4}^{0,4 s+2} \cong \mathbb{Z}_{p} \gamma^{2 s+1}(s \geq 1) \text {. }
\end{aligned}
$$


We have shown that all the generators of the $E_{4}$-page of the spectral sequence survive to $E_{\infty}$. Therefore the LHS spectral collapses at $E_{4}$.

Next we obtain some of the multiplicative relations in the ring $H^{*}(G, \mathbb{Z})$. Since $p \mu=p \nu=p \varepsilon_{1}=p \varepsilon_{2}=0$, it follows that $\mu, \nu, \varepsilon_{1}$ and $\varepsilon_{2}$ are all in the image of the Bockstein map $\delta: H^{*}\left(G, \mathbb{F}_{p}\right) \rightarrow H^{*+1}(G, \mathbb{Z})$. From the structure of the mod- $p$ cohomology ring of $G$ obtained in the previous section, we have that $\Delta\left(Y_{1}\right)=-x_{1} v$ and $\Delta\left(Y_{2}\right)=x_{2} v$. By taking $\mu=\delta\left(Y_{2}\right), \nu=\delta\left(Y_{1}\right), \varepsilon_{1}=\delta\left(Y_{2} v\right)$ and $\varepsilon_{2}=\delta\left(Y_{1} v\right)$, we then have that

$$
\begin{aligned}
& \mu \varepsilon_{1}=\delta\left(Y_{2}\right) \delta\left(Y_{2} v\right)=\delta\left(x_{2} v Y_{2} v\right)=0 \\
& \mu \varepsilon_{2}=\delta\left(Y_{2}\right) \delta\left(Y_{1} v\right)=\delta\left(x_{2} v Y_{1} v\right)=\delta\left(x_{1} v Y_{2} v\right)=-\delta\left(Y_{1}\right) \delta\left(Y_{2} v\right)=-\nu \varepsilon_{1} \\
& \nu \varepsilon_{2}=\delta\left(Y_{1}\right) \delta\left(Y_{1} v\right)=\delta\left(-x_{1} v Y_{1} v\right)=0 .
\end{aligned}
$$

Now let $S^{\prime}=\Sigma_{\imath \geq 0} S_{i}^{\prime}$ be the graded $\mathbb{F}_{p}$-module defined by

$$
S^{\prime}=\mathbb{Z}\left[\alpha^{\prime}, \beta^{\prime}, \xi^{\prime}\right]\left\{1, \mu^{\prime}, \nu^{\prime}, \eta^{\prime}, \varepsilon_{1}^{\prime}, \varepsilon_{2}^{\prime}, \zeta^{\prime}\right\} /\left(R_{7}\right) \otimes_{\mathbb{Z}} \mathbb{F}_{p}
$$

where $\operatorname{deg} \alpha^{\prime}=\operatorname{deg} \beta^{\prime}=2, \operatorname{deg} \mu^{\prime}=\operatorname{deg} \nu^{\prime}=\operatorname{deg} \eta^{\prime}=3, \operatorname{deg} \xi^{\prime}=4, \operatorname{deg} \varepsilon_{i}^{\prime}=5$ $(i=1,2)$, deg $\zeta^{\prime}=6$ and where $R_{7}$ is some term in degree 7 . We have from this and Lemma 4.1 that

$$
\sum_{i \geq 0} t^{2} \operatorname{dim}_{\mathbb{F}_{p}} S_{i}^{\prime}=\frac{1+3 t^{3}+2 t^{5}+t^{6}-t^{7}}{\left(1-t^{2}\right)^{2}\left(1-t^{4}\right)}=Q(t) .
$$

Therefore $H^{*}(G, \mathbb{Z}) \otimes_{\mathbb{Z}} \mathbb{F}_{p} \cong S^{\prime}$ (as vector spaces over $\mathbb{F}_{p}$ ).

We collect the above results in the following theorem:

Theorem 4.9. Let $G=\langle A, B, C| A^{p^{m}}=B^{p^{n}}=C^{p}=[A, C]=[B, C]=1$, $[A, B]=C\rangle$ where $m \geq n \geq 2$. Then the cohomology ring $H^{*}(G, \mathbb{Z})$ is generated by the elements

$$
\alpha, \beta, \mu, \nu, \eta, \xi, \varepsilon_{1}, \varepsilon_{2}, \zeta
$$

where $\operatorname{deg} \alpha=\operatorname{deg} \beta=2, \operatorname{deg} \mu=\operatorname{deg} \nu=\operatorname{deg} \eta=3, \operatorname{deg} \xi=4, \operatorname{deg} \varepsilon_{i}=5 \quad(i=$ $1,2)$ and $\operatorname{deg} \zeta=6$ such that

$$
\begin{aligned}
& p^{m} \alpha=p^{n} \beta=0, \quad p \mu=p \nu=p^{n-1} \eta=0 \\
& p^{2} \xi=0, \quad p \varepsilon_{i}=0(i=1,2), \quad p^{2} \zeta=0
\end{aligned}
$$

The multiplicative relations that are known are 


$$
\begin{gathered}
\mu^{2}=\nu^{2}=\eta^{2}=0, \varepsilon_{i}^{2}=0(i=1,2), \\
\mu \varepsilon_{1}=\nu \varepsilon_{2}=0, \mu \varepsilon_{2}=-\nu \varepsilon_{1} .
\end{gathered}
$$

Moreover, the elements $\alpha, \beta$ and $\xi$ generate a subring of $H^{*}(G, \mathbb{Z})$ such that

$$
H^{*}(G, \mathbb{Z}) \cong \mathbb{Z}[\alpha, \beta, \xi]\left\{1, \mu, \nu, \eta, \varepsilon_{1}, \varepsilon_{2}, \zeta\right\} /\left(R_{7}\right)
$$

where $R_{7}$ is some term in degree 7 .

Remark. The integral cohomology of the group $P_{m, n}$ was also studied in [9]. In his paper, N. Yagita considered the LHS spectral sequence for the extension

$$
1 \rightarrow\langle B, C\rangle \rightarrow P_{m, n} \rightarrow\langle\bar{A}\rangle \rightarrow 1
$$

and showed that the spectral sequence collapses at $E_{2}$ (see Theorem 2.4 in [9]).

The author also notes that some of the integral cohomology ring structure of $P_{2,2}$ for $p \geq 5$ has been obtained in [8] by extending the circle technique of Leary ([3]). In the mod-pcase, the cohomology ring of $P_{m, n}$ obtained in [8, Theorem 6] for the prime $p \geq 5$ is contained in Theorem 3.1 of this paper.

\section{Acknowledgements}

I would like to thank Dr. I. J. Leary for his helpful comments and for pointing out an error in an early version of this paper. The proof given in this paper that $\gamma^{2} \in E_{2}^{0,4}$ survives to $E_{\infty}$ even if $n \geq 3$ is due to a suggestion of Professor N. Yagita. I am indebted to Professor N. Yagita for this and for showing me his corrections to Theorem 2.4 in [9]. Finally, I would also like to thank the referee for some helpful suggestions.

\section{References}

[1] Cárdenas, H. and Lluis, E., On the integral cohomology of a Sylow subgroup of the symmetric group, Comm. Algebra, 18 (1990), 105-134.

[2] Clark, J., Mod 2 cohomology algebra of the group $U_{3}(4)$, Comm. Algebra, 22 (1994). 1419-1434.

[3] Leary, I. J., The cohomology of certain finite groups, Ph. D. Thesis, Cambridge University, 1990.

[4] - The integral cohomology rings of some p-groups, Math. Proc. Camb. Phil. Soc., 110 (1991), 25-32.

[5] - The mod-p cohomology rings of some p-groups, Math. Proc. Camb. Phil. Soc., 112 (1992), 63-75.

[6] - A differential in the Lyndon-Hochschild-Serre spectral sequence, J. Pure and Applied Algebra, 88 (1993), 155-168.

[7] Lewis, G., The integral cohomology rings of groups of order $p^{3}$, Trans. Amer Math. Soc., 132 (1968), 501-529. 
[8] Riesen, J. A., The cohomology ring of a finite p-group, Ph.D. Thesis, Northwestern University, 1993.

[9] Yagita, N., On the dimension of spheres whose product admits a free action by a non-abelian group, Quart. J. Math. Oxford, 2 (1985), 117-127. 